\title{
Impact of gender, depression severity and type of depressive episode on efficacy and safety of escitalopram: an observational study on major depressive disorder patients in southern India
}

Tatiyana Mandal ${ }^{1 *}$ (D), Laxminarayana Kurady Bairy ${ }^{2}$, Podila Satya Venkata Narasimha Sharma ${ }^{3}$ and Vijaya Lakshmi Valaparla ${ }^{4}$

\begin{abstract}
Background: Antidepressant response is a complex trait influenced by clinical, demographic and genetic factors. Objectives: To explore the influences of baseline depression severity, gender and type of depressive episode on efficacy and safety of escitalopram (10-20 mg/day) in South Indian patients with major depressive disorder (MDD).

Methods: The study was conducted on 18-65-year-old patients $(n=151)$ suffering from a first or recurrent episode of MDD with a 17-item Hamilton Depression Rating Scale (HDRS-17) score of $\geq 18$ at baseline. Efficacy assessments were done using HDRS-17, Montgomery-Asberg Depression Rating Scale (MADRS), and Clinical Global Impression (CGI) at baseline and weeks 4, 8 and 12. Patients were monitored for adverse drug reactions (ADRs). Clinical outcomes were compared among various groups based on gender, type of depressive episode (first or recurrent episode) and baseline HDRS-17 scores (moderate depression-score between 17 and 23; severe depression-score $\geq 24$ ).

Results: Among the 148 subjects who completed the 12-week study, 43.9\% and $42.6 \%$ achieved response and remission, respectively. The decline in HDRS-17 and MADRS scores from baseline was significant $(p$ value $<0.05)$ at all follow-up visits and a similar pattern was seen with CGI. Efficacy outcomes were better in the moderate baseline depression group compared with severe depression. There were no associations of efficacy with gender and type of depressive episode. A total of 247 adverse drug reactions (ADR) were reported and 119 (80.41\%) subjects experienced at least one ADR during the study period. No serious ADR was reported. Male patients experienced more ADRs compared with females. The safety profile of escitalopram was similar across various groups based on baseline depression severity and type of depressive episode.
\end{abstract}

Conclusion: The study revealed that escitalopram is efficacious in south Indian MDD patients with a favourable safety profile. The efficacy was influenced by baseline depression severity whereas more ADRs were reported by male patients.

Keywords: Depressive disorder, major, Efficacy, Escitalopram, Indians, south, Safety

\footnotetext{
*Correspondence: tatiyana.mandal@gmail.com;

tatiyana.mandal@manipal.edu

'Department of Pharmacology, Melaka Manipal Medical College (Manipal

Campus), Manipal Academy of Higher Education (MAHE), Manipal, Karnataka

576104 , India

Full list of author information is available at the end of the article
}

\section{Springer Open}

(c) The Author(s). 2021 Open Access This article is licensed under a Creative Commons Attribution 4.0 International License, which permits use, sharing, adaptation, distribution and reproduction in any medium or format, as long as you give appropriate credit to the original author(s) and the source, provide a link to the Creative Commons licence, and indicate if changes were made. The images or other third party material in this article are included in the article's Creative Commons licence, unless indicated otherwise in a credit line to the material. If material is not included in the article's Creative Commons licence and your intended use is not permitted by statutory regulation or exceeds the permitted use, you will need to obtain permission directly from the copyright holder. To view a copy of this licence, visit http://creativecommons.org/licenses/by/4.0/. 


\section{Introduction}

According to the World Health Organization, globally approximately 264 million people are affected by major depressive disorder (MDD) [1]. In India, the National Mental Health Survey has estimated that at any given point, nearly 23 million adults would require care for depressive disorders [2]. The burden of MDD is further amplified by the high suicide rate (up to 15\%), stressrelated complications and associated deleterious effects on the cardiovascular system [3, 4]. Statistical data suggests that by 2030 , unipolar depression will become the second highest contributor to the global disease burden [5].

Over the last few decades, SSRIs have emerged as the first line of treatment for depressive disorders because of their effectiveness and relatively low toxicity $[6,7]$. Various comparative trials have suggested that escitalopram, the S-enantiomer of citalopram has better efficacy and tolerability in MDD patients compared with other SSRIs [8-10]. Escitalopram is known to produce only mild and transient adverse drug reactions (ADR) [9]. The most common ADRs reported with escitalopram therapy were insomnia, fatigue, somnolence, nausea, headache, dry mouth, excessive sweating and sexual dysfunction $[9,11]$. In India, studies have found escitalopram being frequently prescribed as a first-line antidepressant [12].

There is a considerable debate on the efficacy and tolerability of SSRIs in MDD patients. A substantial proportion (up to $30-50 \%$ ) of depressed patients shows insufficient response to SSRI treatment [13]. Sixty to seventy percent of MDD patients are presented with treatment-resistant depression (TRD) which is an important contributor of significant morbidity and mortality associated with MDD [14]. Several socio-demographic factors that predispose patients to TRD include old age, patients with poor economic resources, lack of education, poor social support and family networks and lower function and quality of life [15]. Specific clinical features of the MDD episode such as longer duration and recurrent episode, higher severity, high suicidal risk are potentially predictive indicators of TRD [16]. Non-adherence to antidepressant therapy is another important reason for treatment failure [17]. Many patients fail to adhere to antidepressant therapy because of adverse drug reactions (ADR) [18]. Psychiatric and somatic comorbidities (anxiety or panic disorder, substance abuse, higher level of objective stress, personality disorders, neurodegenerative or autoimmune diseases etc.) are also associated with treatment non-response and higher risk of TRD [19]. Further, genetic variants within the serotonin transporter-serotonin receptors and antidepressant metabolizing enzymes or genes involved in neurodevelopment have been found to modulate the risk of TRD [20]. Since antidepressant response is a complex trait influenced by clinical, environmental, genetic and drug-associated factors, treatment response and ADRs might vary in different populations [21]. Several studies have also reported an association between antidepressant response and baseline disease severity [22].

Presently in routine clinical practice, psychiatrists have a wide choice of antidepressants. Therefore, it is essential to create a population-specific evidence base for efficacy and tolerability profile of antidepressants which will help clinicians to make the best choice for individual patients. Further, identification of variables predicting antidepressant treatment outcomes has become one of the major focuses of current scientific research because this might hold the potential to transform the otherwise lengthy process of antidepressant selection for individual patients and thereby might reduce the overall burden and financial loss. There is an inadequacy of data on escitalopram treatment response and the plausible influence of disease severity in modulating this, specifically in a south Indian population. Data regarding influence of other patient and disease-related factors on treatment response is also rare. Therefore, we intended to explore the treatment response and ADR profile of escitalopram in South Indian MDD patients. The influences of the baseline severity of depression, gender and type of depressive episode on treatment response and safety profile were also assessed.

\section{Materials and methods}

This 12-week, prospective, open-label, observational study was conducted at the Department of Psychiatry, Kasturba Medical College Hospital, Manipal, India. The study was approved by the Institutional Ethics Committee of Kasturba Hospital, Manipal (Registration no. ECR/ 146/Inst/KA/2013; Study approval number: IEC 317/ 2013). Written informed consent was obtained from all the participants after explaining the full procedure.

\section{Subjects}

The study subjects were patients who were diagnosed with a current episode of unipolar depression and were on escitalopram monotherapy. The diagnosis was verified by DSM-IV (Diagnostic and Statistical Manual of Mental Disorders, Fourth Edition) criteria [23]. A thorough neuro-psychiatric interview was conducted during screening. After the initial diagnosis, the treatment regimen was decided for all MDD patients and it was solely at the discretion of the treating psychiatrist who was not involved in the study. Later, only those MDD patients prescribed with escitalopram monotherapy were screened for the exclusion and inclusion criteria. Patients were recruited for the study if they had fulfilled these criteria and had given consent to participate in our study. The study had the following inclusion criteria: (a) Patients having baseline 17-item Hamilton 
Depression Rating Scale (HDRS-17) score of 18 and above, (b) South Indian ethnicity, (c) Either gender, (d) Age between 18 and 65 years, (e) Patients with first episode or recurrent episode of MDD. Patients were excluded if they were diagnosed with other co-morbid mental illnesses such as schizophrenia, bipolar disorder, schizoaffective disorders, alcohol/drug abuse-related disorder or dementia within 12 months from week 0 . Additional exclusion criteria included patients with significant suicide risk, liver or renal impairment, unstable serious illness, contraindications to escitalopram, pregnant or lactating women. Patients were enrolled from both inpatient and outpatient settings. Ethnicity of the patient was verified by taking a detailed family history. All study subjects were treated with escitalopram once daily at a flexible dose of 10-20 mg. Treatment was initiated with a once-daily dosage of $10 \mathrm{mg}$ escitalopram. If adequate response was not observed during the follow-up visits, doses were titrated up to $20 \mathrm{mg}$ per day. Patients were not allowed to take any other psychotropic medications except zolpidem or zopiclone or clonazepam for insomnia and/or anxiety. However, patients were allowed to take medications for general illnesses like diabetes, hypertension etc. provided those are not contraindicated with escitalopram. Concomitant medications and dosages were recorded. Adherence to medication was ascertained when patients came for follow-up by patients' self-report and also by counting unused drugs. Any patient who had not taken $20 \%$ of the prescribed drug was considered non-compliant and excluded from the study. The study subjects received the standard treatment throughout the study period and the treatment regimen was supervised independently by the psychiatrist treating the patient. This study received no specific grant from any funding agency and the cost of the treatment was borne solely by the patients.

\section{Efficacy assessment}

HDRS-17, Montgomery-Asberg Depression Rating Scale (MADRS) and Clinical Global Impression (CGI) were used for efficacy assessments. The primary efficacy outcomes were the remission rate and response rate. Criteria for remission were MADRS score less than 12 and HDRS-17 score less than 8 . At least a $50 \%$ decline in HDRS 17 and MADRS total scores from baseline was considered as treatment response. Secondary efficacy outcomes were changes in HDRS-17, MADRS and CGI from baseline to week 12. The Columbia-Suicide Severity Rating Scale was used to assess the suicidal tendency of the patients. Based on the baseline HDRS-17 scores, the subjects were categorized into two groups: group 1 (moderate depression)-HDRS-17 score between 17 and $23(n=91)$, group 2 (severe depression)-HDRS-17 score $\geq 24(n=57)$. Grouping was also done based on gender (male and female) and type of depressive episode (first or recurrent episode). Clinical outcomes were compared among various groups.

\section{Safety assessment}

After patient recruitment, a baseline review was done to identify any symptoms which were present prior to the drug therapy. The frequency and type of suspected adverse drug reactions (ADRs) were noted. Inpatients were monitored for ADR throughout their hospital stay while outpatients were monitored during their subsequent visits to the outpatient department over a period of 12 weeks. Weight and other vital signs were also monitored each time. Causality assessments of ADRs were done using the Naranjo ADR Probability Scale [24]. The scale consists of a list of 10 weighed questions addressing various aspects of the suspected ADR such as temporal association of drug administration and ADR occurrence, drug levels in the body, alternative causes for the ADR, change in severity with dose changes, previous patient experience with the same drug, effect of drug discontinuation, specific antagonist or drug rechallenge and availability of objective evidence. These questions can be answered with 'yes', 'no' or 'do not know' and each answer is attached to prefixed numerical scores which result in a cumulative value. The ADR is assigned to one of the following probability categories based on the $\mathrm{cu}-$ mulative score: definite $(\geq 9)$, probable $(5-8)$, possible (1-4) and doubtful (0). A modified Hartwig and Siegel Severity Assessment Scale was used to assess the severity of ADRs [25]. The scale classifies the severity of suspected ADR as mild, moderate and severe and there are 7 levels. Mild-level 1 severity requires no change in drug therapy whereas mild-level 2 requires the suspected drug to be withheld, discontinued or changed. For moderate-level 3, along with change of drug therapy, administration of an antidote or other therapeutic agents is required. If there is an increase in the length of hospital stay by at least one day, the ADR belongs to the moderate level 4(a) category. The ADR is classified as moderate-level 4(b) if the patient is hospitalized due to the ADR. Severe-level 5 ADRs warrant intensive care for the patient. If the ADR has caused permanent harm to the patient, it is a severe-level 6 ADR. The most severe ADR, i.e. level 7 ADRs, are those which lead to the death of the patient either directly or indirectly.

\section{Statistical analysis}

Student's $t$ test and Chi-square test were used to analyse quantitative and qualitative data, respectively. The changes in treatment response over time was analysed by one-way ANOVA followed by Bonferroni's post hoc test. The other efficacy outcomes and ADR-related assessments were analysed descriptively. SPSS 19.0 was 
used for statistical analysis and $p$ value $<0.05$ was considered statistically significant.

\section{Results}

Demographic and clinical characteristics of study subjects at baseline

A total of 151 depressive patients were recruited in the study among which 3 patients were excluded from the study as they did not participate in the follow-up evaluation. All the subjects were of South Indian ethnicity and were from states of Karnataka, Kerala and Andhra Pradesh. The demographic and clinical characteristics of the subjects at baseline are presented in Table 1. 15.5\% of patients had suicidal ideation at baseline, but it decreased to $6.08 \%$ at the end of 12 weeks. The intensity of ideation was least severe as assessed by the Columbia Suicide Severity Rating Scale and these patients did not require any additional psychotropic medications. None of the subjects were found to have suicidal tendencies during the study period. Escitalopram therapy was started with an initial dose of $10 \mathrm{mg}$ once daily for all patients. Later, the escitalopram dose was adjusted to a maximum of $20 \mathrm{mg} /$ day for 124 (83.78\%) patients during the treatment period and the rest were maintained with the initial dose till the completion of the study. At least one concomitant drug was taken by $47(31.8 \%)$ patients. The most common concomitant drug prescribed to the patients was clonazepam (7.4\%) for insomnia.

\section{Efficacy outcomes}

\section{Remission and response rate}

According to the criteria for response, at the end of week 12, out of 148 study subjects, 83 (56.08\%) patients

Table 1 Baseline demographic and clinical characteristics of patients

\begin{tabular}{ll}
\hline Characteristic, $\boldsymbol{N}=\mathbf{1 4 8}^{*}$ & \\
\hline Female, $n$ (\%) & $87(58.8)$ \\
Male, $n$ (\%) & $61(41.2)$ \\
Ethnicity & South Indian \\
Age in years, mean \pm SD & $42.2 \pm 10.7$ \\
Patients experiencing first episode, $n$ (\%) & $102(68.9)$ \\
Patients experiencing recurrent episode $n(\%)$, & $46(31.1)$ \\
HDRS-17 score, mean \pm SD & $22.56 \pm 3.22$ \\
MADRS score, mean \pm SD & $33.22 \pm 4.23$ \\
CGI-S score, $n$ (\%) & \\
$4=$ Moderately ill & $47(31.5)$ \\
$5=$ Markedly ill & $52(34.9)$ \\
$6=$ Severely ill & $49(32.9)$ \\
Patients having suicidal ideation, $n(\%)$ & $23(15.5)$ \\
Patients having suicidal tendency, $n(\%)$ & $0(0)$ \\
\hline
\end{tabular}

${ }^{*} \mathrm{~N}$ - Total number of study subjects were non-responders and $65(43.9 \%)$ patients were responders. Sixty-three (96.9\%) patients fulfilled the criteria of remission. Figure 1 shows the week-wise remission and response rates in the total study population and patients with different baseline depression levels. Response and remission rates were higher in group 1 compared with that of group 2 at weeks 4,8 and 12 (Fig. 1). However, a comparison of the number of responders and remitters among studied groups (group 1 and group 2) using Chi-square test or Fisher's exact test revealed that the association between baseline depression severity and treatment response and remission was not statistically significant.

\section{Changes in HDRS-17, MADRS and CGI scores}

Figures 2 and 3 represent the change in mean HDRS-17 scores and MADRS scores during escitalopram therapy in different groups. The decline in HDRS-17 and MADR $\mathrm{S}$ scores in the total study population was significant ( $p$ value 0.0001 ) at week 4 and was sustained till week 12 . Changes in CGI-severity and CGI-improvement ratings are given in Table 2. A similar pattern of improvement was seen with CGI-S and CGI-I scores compared with that seen with the HDRS-17 and MADRS total scores.

Group-wise analysis revealed that, in both groups, HDRS-17 and MADRS scores were significantly reduced at different time-points compared with baseline. The decline in HDRS-17 and MADRS scores was significantly ( $p$ value 0.0001 ) higher in group 1 at weeks 4,8 and 12 compared with group 2 (Figs. 2 and 3) which was indicative of better treatment response in patients with lower baseline depression severity.

\section{Relationship of efficacy with gender and type of depressive episode}

Efficacy outcomes were similar across different genders $(p>05)$. No significant difference $(p>.05)$ was observed in efficacy outcomes between patients experiencing a first episode and patients experiencing a recurrent episode.

\section{Safety and tolerability}

Out of 148 study subjects, 119 (80.41\%) subjects experienced at least 1 adverse drug reaction (ADR) during the study period. The maximum number of patients $(66.9 \%)$ experienced ADR at week 4 followed by week 8 (29.1\%) and week 12 (7.4\%). Most of the ADRs (except insomnia) were mild requiring no change of treatment or drug withdrawal. Insomnia was moderate in $11(7.4 \%)$ patients and was treated with clonazepam. There were no serious ADRs reported. Causality analysis revealed that 196 (79.4\%) ADRs were probable whereas 51 (34.6\%) ADRs were possible in nature. Doubtful ADRs were excluded from analysis. A total of 247 ADRs were reported during 


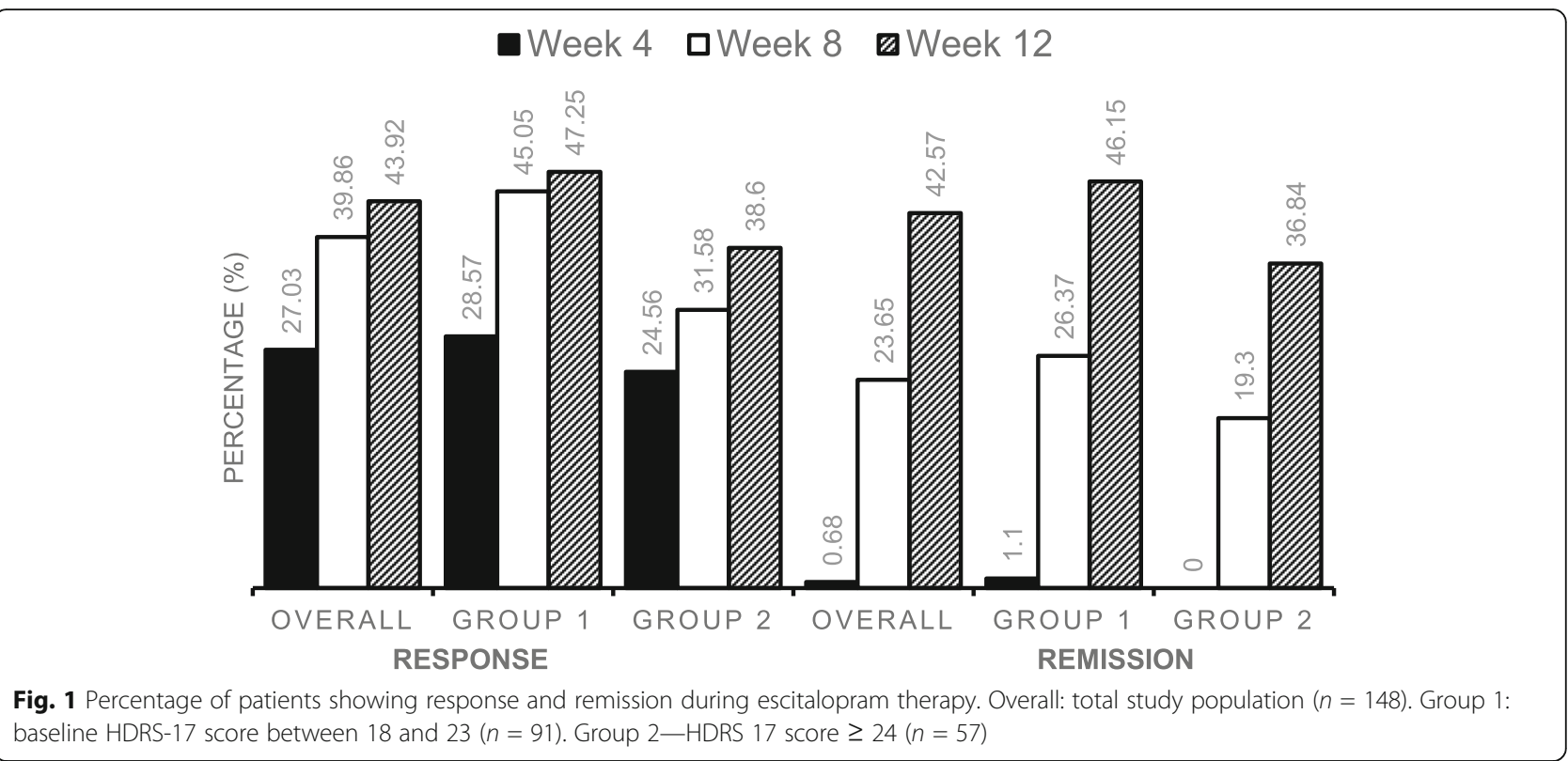

the 12-week study period. $67.2 \%$ of ADRs were reported at the 4th week of treatment followed by the 8th week $(26.3 \%)$ and the 12th week (6.5\%). Escitalopram was well tolerated by the study subjects and treatment withdrawal due to ADRs was not required for any patients. The common ADRs observed during the study period are summarized in Table 3. There were no significant differences in the proportion of patients experiencing ADRs among different groups based on baseline disease severity, gender and type of depressive episode. The mean number of ADRs was similar across patients having different baseline depression severity and types of depressive episodes. The mean number of ADRs experienced by male patients (mean \pm SEM-1.97 \pm 0.19 ) was significantly higher ( $p$ value: .025 )

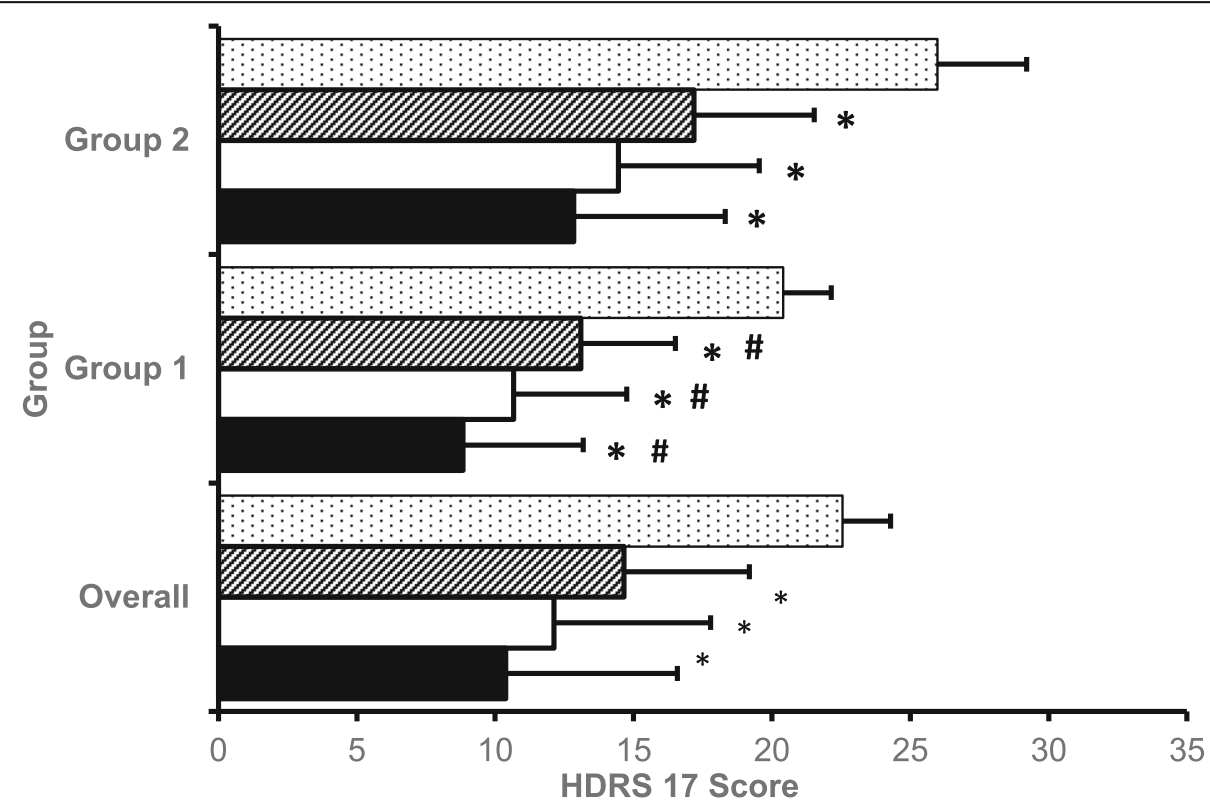

๑Week 0 घWeek 4 W.Week 8 WWeek 12

Fig. 2 Mean change in HDRS-17 scores with escitalopram therapy. Group 1: baseline HDRS-17 score between 18 and 23 ( $n=91$ ). Group 2 - HDRS-17 score $\geq 24(n=57) .{ }^{*} p$ value $<0.05$ HDRS-17 scores at weeks 4,8 and 12 compared with week $0 .{ }^{*} p$ value $<0.05$ compared with respective scores in group 2 


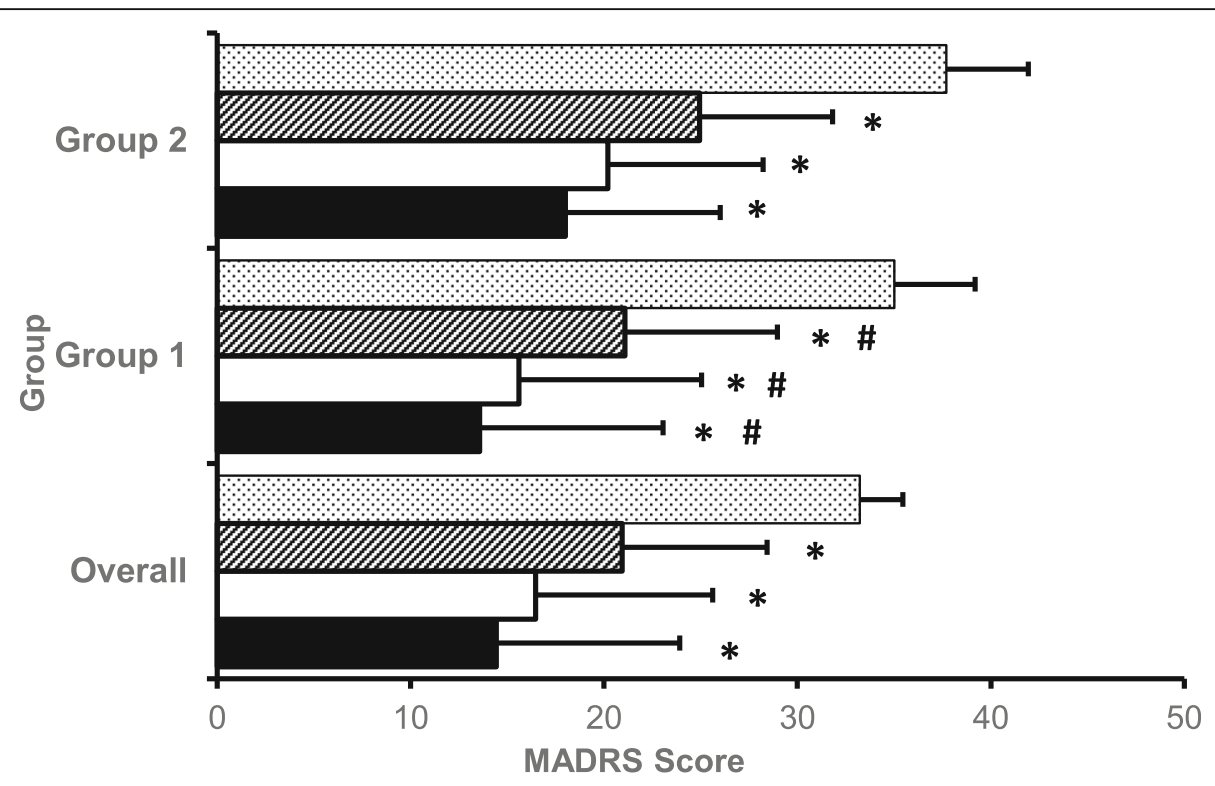

W. Week 0 -Week 4 aWeek 8 Wheek 12

Fig. 3 Mean change in MADRS scores with escitalopram therapy. Group 1: baseline HDRS-17 score between 18 and $23(n=91)$. Group 2-HDRS17 score $\geq 24(n=57) .{ }^{*} p$ value $<0.05$ HDRS-17 scores at weeks 4,8 and 12 compared with week $0 .{ }^{*} p$ value $<0.05$ compared with respective scores in group 2

compared with female patients (mean \pm SEM-1.46 \pm 0.13 ) in our study population. The more prevalent ADRs among males (reported by $\geq 4 \%$ of male patients) as compared with female patients were nausea, diarrhoea, fatigue, constipation, sweating, drowsiness and abdominal pain. Sexual dysfunction was reported exclusively by male patients.

\section{Discussion}

Perusal of the data collected shows that escitalopram was effective in the treatment of MDD patients and it was well tolerated. At the end of 12 weeks, a response rate of $43.9 \%$ and a remission rate of $42.6 \%$ were achieved in the present study. The pattern of treatment

Table 2 Changes of clinical global impression during escitalopram therapy ( $\left.N=148^{*}\right)$

\begin{tabular}{|c|c|c|c|}
\hline$\overline{C G I}$ & Week 4, n (\%) & Week 8, n (\%) & Week 12, $n(\%)$ \\
\hline \multicolumn{4}{|l|}{ CGI-S (severity) } \\
\hline Normal & $0(0)$ & $4(2.70)$ & $36(24.32)$ \\
\hline Marginally ill & $1(0.68)$ & $27(18.24)$ & $24(16.22)$ \\
\hline Mildly ill & $93(62.84)$ & $79(53.38)$ & $61(41.22)$ \\
\hline Moderately ill & $24(16.22)$ & $23(15.54)$ & $16(10.81)$ \\
\hline Markedly ill & $27(18.24)$ & $15(10.14)$ & $11(7.43)$ \\
\hline Severely ill & $3(2.03)$ & $0(0)$ & $0(0)$ \\
\hline Most extremely ill & $0(0)$ & $0(0)$ & $0(0)$ \\
\hline \multicolumn{4}{|l|}{ CGI-I (improvement) } \\
\hline Very much improved & $2(1.35)$ & $28(18.92)$ & $54(36.49)$ \\
\hline Much improved & $57(38.51)$ & $52(35.14)$ & $36(24.32)$ \\
\hline Minimally improved & $64(43.24)$ & $60(40.54)$ & $53(35.81)$ \\
\hline No change & $25(16.89)$ & $8(5.41)$ & $5(3.38)$ \\
\hline Minimally worse & $0(0)$ & $0(0)$ & $0(0)$ \\
\hline Much worse & $0(0)$ & $0(0)$ & $0(0)$ \\
\hline Very much worse & $0(0)$ & $0(0)$ & $0(0)$ \\
\hline
\end{tabular}

${ }^{*} \mathrm{~N}$ - Total number of study subjects 
Table 3 Summary of adverse drug reactions (ADRs) seen in $\geq$ $3 \%$ of patients $(N=148)$

\begin{tabular}{ll}
\hline ADR & $\boldsymbol{n}$ (\%) \\
\hline Nausea & $29(19.6)$ \\
Headache & $26(17.6)$ \\
Diarrhoea & $22(14.9)$ \\
Dry mouth & $20(13.5)$ \\
Decreased appetite & $18(12.2)$ \\
Insomnia & $16(10.8)$ \\
Fatigue & $14(9.5)$ \\
Constipation & $14(9.5)$ \\
Dizziness & $12(8.1)$ \\
Tremors & $9(6.1)$ \\
Vomiting & $9(6.1)$ \\
Sweating & $8(5.4)$ \\
Drowsiness & $8(5.4)$ \\
Abdominal pain & $6(4.1)$ \\
Anxiety & $5(3.4)$ \\
Sexual dysfunction & \\
\hline
\end{tabular}

$N$, total study population; $n$, number of patients reporting ADR

a Percentage is calculated out of male patients only $(n=61)$

response assessed by HDRS-17, MADRS and CGI scores were consistent which is suggestive of minimal bias, thus establishes the clinical relevance of treatment response and remission. The response and remission rate observed in our study is lower compared with studies reported in the Indian population. These studies reported a response rate and remission rate of more than $70 \%$ and $60 \%$ respectively at week $8[26,27]$. Another multicenter trial of escitalopram in MDD patients found $80 \%$ response and remission rates at week 8 [28]. A pooled analysis of four Chinese clinical trials reported a $68.4 \%$ response rate and a $48.4 \%$ remission rate at the end of 7 weeks [29]. The differences in response and remission rates found in our study could be attributed to variability in study design, sample size, study population, duration of treatment and type of depressive disease.

In our study, the mean changes in HDRS 17 and MADRS were observed in week 4 and there was a steady decline in scores in the next follow-up visits. The changes were statistically significant at each time point compared with baseline. The mean decline in HDRS-17 and MADRS scores at 12 weeks was -10.4 and -18.8 respectively. At the end of 8 weeks, the mean decline of HDRS-17 and MADRS was -12.2 and -16.8 respectively. This finding is consistent with several comparative studies of escitalopram versus other SSRIs or placebo in MDD patients [30, 31]. However, one Indian study reported a higher decline in MADRS score (- 20.8) compared with our study at week 8 [26]. This could be due to the higher proportion of non-responders in our study population. A 24-week study reported a much higher decline (- 26.2) in MADRS scores from baseline [11]. Studies have found that with continued treatment, patients not showing response at week 8 had achieved remission at week $24[11,30]$. In the present study, 36.5\% of patients showed very much improvement after 12 weeks of treatment according to the CGI Improvement Scale, whereas $18.9 \%$ of patients showed very much improvement at 8 weeks of treatment. Therefore, the duration of escitalopram therapy can be considered as an important determinant of treatment remission and severely depressed patients might require a longer duration of treatment for achieving remission. Considering a substantial percentage (32.9\%) of severely depressed patients at baseline, we might expect an increase in remission rate if the study would have continued for a longer duration. Moreover, most of the responders at week 8 achieved remission at the end of 12 weeks. Therefore, the treatment response observed at week 8 may be used to predict remission in MDD patients.

We have found that patients with baseline moderate depression (HDRS-17 score 17-23) showed better response and remission rate compared with patients with severe depression (HDRS-17 score $\geq 24$ ). The decline in HDRS-17 and MADRS scores was also significantly higher in moderate depression. These findings are in line with four trials of escitalopram in MDD patients in the Chinese population [29]. However, several studies reported that escitalopram showed better treatment response compared with other SSRIs in patients having severe depression at baseline as well [10, 32]. However, the absence of a placebo or another treatment group for comparison of treatment efficacy can be considered as a limitation of this study.

In our study, remission was achieved at a dose of 10 $20 \mathrm{mg}$ of escitalopram which is consistent with several trials of escitalopram [26, 30]. Out of 148 patients, dose was increased to $20 \mathrm{mg} /$ day for 124 patients which include all non-responders $(n=83)$ and 41 (out of 65) responders during the 12-week treatment period. Since dose was increased for more than $80 \%$ of study subjects, we have not done any dose-wise analysis of treatment response and remission. An earlier study suggests that escalation of escitalopram dose beyond $20 \mathrm{mg}$ might have a beneficial effect on treatment response [33]. However, we could not explore this effect in our study as the escitalopram dose was maintained at a maximum of $20 \mathrm{mg}$.

Sub-group analysis of treatment response revealed that efficacy outcomes were similar in all patients irrespective of their gender or type of depressive episode. Although we did not find any statistically significant associations of these factors with treatment response in our study, but there are conflicting reports in the literature [29, 34, 35]. Hence, future studies are needed to substantiate these 
reports. Another limitation of our study is that we have not investigated the effect of other factors such as age, duration of depressive episode and concomitant anxiety symptoms which are also known to influence the treatment response [11, 36, 37]. Moreover, the presence of suicidality can attenuate the clinical response to antidepressant treatment, independently of clinical confounders or the type of antidepressant [38]. A systematic review of several randomized clinical trials raised concerns regarding the increase in suicidality in young psychiatric patients receiving SSRIs [39], whereas several pharmacoepidemiological studies have demonstrated the protective effects of antidepressants with respect to suicidal behaviour in depressive patients [40]. This controversial context demands clinical trials to be designed specifically for depressed patients at significant risk for suicide. In our study, $15.5 \%$ of study subjects were found to have suicidal ideation at baseline and we had observed a decline in suicidal ideation at the end of 12 weeks with escitalopram treatment. The intensity of the ideation was found to be the least severe as assessed by the Columbia Suicide Severity Rating Scale. None of the study subjects were found to have suicidal tendencies at baseline or developed it during the study period. However, it is important to note that we had recruited only those patients with suicidal ideation who do not require any other psychotropic medications and can be managed by escitalopram itself. Patients with significant suicide risk such as those with other co-morbid psychiatric or medical conditions, severe somatic illness, adverse life situations, previous suicide attempts, family history of suicide or psychosocial risk factors were excluded from our study as the management of these patients include close monitoring, psychotherapy and use of other psychotropic medications (lithium, antipsychotics, anxiolytics) along with antidepressants [41]. Hence, from our study findings, we could not establish the therapeutic efficiency of escitalopram in patients with significant suicide risk.

In this study, adverse drug reactions to escitalopram doses of 10-20 mg were mild to moderate in nature and tolerable. Serious ADRs and unexpected safety issues were not reported. The common side effects were related to the gastrointestinal system. In addition, headache, insomnia and fatigue were also commonly reported. No adverse cardiac outcomes were observed in our study, though there are some conflicting reports regarding cardiac safety of escitalopram [42, 43]. Most of the ADRs did not require any treatment and were subsided or reduced on their own over few days or weeks. Majority of the adverse effects were reported during the first 4 weeks of therapy. These findings are consistent with the earlier studies of escitalopram in patients with MDD in India and other Asian countries [9, 11, 26-28]. There was no patient withdrawal in our study due to ADR. This finding is also in line with various studies where escitalopram was reported to have lower drop-out cases due to ADR compared with other SSRIs [8, 44]. Our study also demonstrated that the frequency of ADR was not associated with baseline disease severity, gender and type of depressive episode.

Escitalopram is the S-enantiomer of citalopram having 30 to 40 times more potency compared with the Renantiomer in binding to serotonin transporter [45]. This 'chiral-switching' allowed escitalopram to have a more selective action on the serotonin transporter than other SSRIs [46]. Therefore, it can inhibit the serotonin reuptake more effectively resulting in better treatment efficacy. The low affinity of escitalopram towards cholinergic, alpha-adrenergic and histaminergic receptors might account for its better safety and tolerability profile [47]. Thus, escitalopram is presumed to be highly efficacious and safe for the treatment of MDD patients. This presumption is supported by the results of our study. However, these results cannot be extrapolated for all MDD patients because patients with co-morbid psychiatric disorders, anxiety disorders and high suicide risk were excluded from our study. Therefore, our study population may not be fully exemplary of MDD patient population. This could be another limitation of our study. Further a multi-centric, large-scale, longer duration, prospective, comparative study in a variety of patient populations will be required to establish the efficacy and safety of escitalopram for the treatment of MDD.

Robust scientific evidence suggests that genetic polymorphisms have a crucial role in determining the antidepressant response and adverse effects of escitalopram in different populations [48-50]. Considering the south Indian population to have a distinct genetic makeup [51], we explored the treatment response and ADR profile of escitalopram exclusively in MDD patients of South Indian origin. We can infer that the lower response and remission rate with escitalopram observed in our study population compared with other populations could be due to the genotypic differences. We cannot confirm this at this stage due to a lack of pharmacokinetic and genotypic data. Further large-scale genetic association studies of several pharmacokinetic and pharmacodynamics candidate genes with treatment response will be required to prove this hypothesis.

\section{Conclusion}

Based on our study results, we can conclude that escitalopram is an efficacious SSRI for the treatment of MDD in South Indian patients with favourable safety and tolerability profile. It was also observed that the treatment response of escitalopram can be influenced by baseline depression severity. This prospective surveillance study provides a representative data of treatment response and ADR profile of escitalopram likely to be encountered in 
South Indian patients with MDD. Our findings would be useful to clinicians in rational prescribing by providing an estimate of efficacy at multiple time points for comparison. This study data might serve as an evidence base to assist clinicians in predicting clinical outcomes. It also might help in the early detection of ADRs and their subsequent management thereby improving the overall health outcomes and quality of life of patients with major depressive disorder.

\section{Abbreviations \\ MDD: Major depressive disorder; SSRI: Selective serotonin reuptake inhibitor; ADR: Adverse drug reaction; DSM IV: Diagnostic and Statistical Manual of Mental Disorders, Fourth Edition; HDRS-17: 17-item Hamilton Depression Rating Scale; MADRS: Montgomery-Asberg Depression Rating Scale; \\ CGI: Clinical Global Impression; CGI-S: Clinical Global Impression-Severity; CGI- I: Clinical Global Impression-Improvement}

\section{Acknowledgements}

We sincerely acknowledge the assistance provided by Dr. Amritavarshini R and Dr. Linda Thomas from the Department of Psychiatry, KMC, Manipal, during data collection. We are thankful to Dr. Smita Shenoy, Department of Pharmacology, KMC, Manipal, and Dr. Ravindra Munoli, Department of Psychiatry, KMC, Manipal, for their valuable inputs on the study.

\section{Authors' contributions}

TM was involved in study design, data collection, analysis and interpretation and preparation of the manuscript. LKB, PSVNS and VLV were involved in study design, data analysis and interpretation and critical revision of the manuscript. The author(s) read and approved the final manuscript.

\section{Funding}

This research received no specific grant from any funding agency in the public, commercial, or not-for-profit sectors.

\section{Availability of data and materials}

The datasets used and/or analysed during the current study are available from the corresponding author on reasonable request.

\section{Declarations}

\section{Ethics approval and consent to participate}

The study was approved by the Institutional Ethics Committee of Kasturba Hospital, Manipal (Registration no. ECR/146/Inst/KA/2013), and the approval number of our study is IEC 317/2013. Written informed consent was obtained from all the participants after explaining the full procedure.

\section{Consent for publication}

Not applicable

\section{Competing interests}

The authors declare that they have no competing interests

\section{Author details}

'Department of Pharmacology, Melaka Manipal Medical College (Manipal Campus), Manipal Academy of Higher Education (MAHE), Manipal, Karnataka 576 104, India. ${ }^{2}$ Department of Pharmacology, RAK College of Medical Sciences, RAK Medical \& Health Sciences University, Ras Al Khaimah, UAE. ${ }^{3}$ Department of Psychiatry, Kasturba Medical College, Manipal Academy of Higher Education (MAHE), Manipal, Karnataka 576 104, India. ${ }^{4}$ Department of Neurology, University of Texas Medical Branch, Galveston, TX, USA.

\section{Received: 5 August 2020 Accepted: 22 March 2021}

Published online: 31 March 2021

\section{References}

1. World Health Report 2004: changing history. WHO; 2004.

2. Arvind BA, Gururaj G, Loganathan S, Amudhan S, Varghese M, Benegal V, et al. Prevalence and socioeconomic impact of depressive disorders in India:
Multisite population-based cross-sectional study. BMJ Open. 2019;9(6): e027250. https://doi.org/10.1136/bmjopen-2018-027250.

3. Dhar AK, Barton DA. Depression and the link with cardiovascular disease. Front Psychiatry. 2016;7(MAR):1.

4. Xin LM, Chen L, Su YA, Yang FD, Wang G, Fang YR, et al. Risk factors for recent suicide attempts in major depressive disorder patients in China: results from a National Study. Front Psychiatry. 2018;9:300. https://doi.org/1 0.3389/fpsyt.2018.00300.

5. Mathers $C D$, Loncar D. Projections of global mortality and burden of disease from 2002 to 2030. PLoS Med. 2006:3(11):2011-30.

6. Preskorn SH. Clinically relevant pharmacology of selective serotonin reuptake inhibitors. An overview with emphasis on pharmacokinetics and effects on oxidative drug metabolism. Clin Pharmacokinet. 1997; 32(Suppl 1):1-21.

7. Anderson IM, Ferrier IN, Baldwin RC, Cowen PJ, Howard L, Lewis G, et al. Evidence-based guidelines for treating depressive disorders with antidepressants: a revision of the 2000 British Association for Psychopharmacology guidelines. J Psychopharmacol. 2008;22(4):343-96. https://doi.org/10.1177/0269881107088441.

8. Lalit V, Appaya PM, Hegde RP, Mital AK, Mittal S, Nagpal R, et al. Escitalopram versus citalopram and sertraline: a double-blind controlled, multi-centric trial in Indian patients with unipolar major depression. Indian J Psychiatry. 2004;46(4):333-41.

9. Kirino E. Escitalopram for the management of major depressive disorder: a review of its efficacy, safety, and patient acceptability. Patient Prefer Adherence. 2012;6:853-61. https://doi.org/10.2147/PPA.S22495.

10. Boulenger JP, Huusom AKT, Florea I, Bæmadraskdal T, Sarchiapone M. A comparative study of the efficacy of long-term treatment with escitalopram and paroxetine in severely depressed patients. Curr Med Res Opin. 2006; 22(7):1331-41. https://doi.org/10.1185/030079906X115513.

11. Jiang $K$, Li L, Wang $X$, Fang $M$, Shi J, Cao Q, et al. Efficacy and tolerability of escitalopram in treatment of major depressive disorder with anxiety symptoms: A 24-week, open-label, prospective study in Chinese population. Neuropsychiatr Dis Treat. 2017;13:515-26. https:// doi.org/10.2147/NDT.S120190.

12. Grover S, Avasth A, Kalita K, Dalal P, Rao G, Chadda R, et al. IPS multicentric study: antidepressant prescription patterns. Indian J Psychiatry. 2013;55(1): 41-5. https://doi.org/10.4103/0019-5545.105503.

13. Steimer W. Pharmacogenetics and psychoactive drug therapy: ready for the patient? Ther Drug Monit. 2010;32(4):381-6. https://doi.org/10.1097/FTD. ob013e3181e1a78d

14. Fava M. Diagnosis and definition of treatment-resistant depression. Biol Psychiatry. 2003;53(8):649-59. https://doi.org/10.1016/50006-3223(03 )00231-2.

15. Balestri M, Calati R, Souery D, Kautzky A, Kasper S, Montgomery S, et al. Socio-demographic and clinical predictors of treatment resistant depression: a prospective European multicenter study. J Affect Disord. 2016;189:224-32. https://doi.org/10.1016/j.jad.2015.09.033.

16. Souery D, Oswald P, Massat I, Bailer U, Bollen J, Demyttenaere K, et al. Clinical factors associated with treatment resistance in major depressive disorder: results from a European multicenter study. J Clin Psychiatry. 2007; 68(7):1062-70. https://doi.org/10.4088/JCP.v68n0713.

17. Bull SA, Hu XH, Hunkeler EM, Lee JY, Ming EE, Markson LE, et al. Discontinuation of use and switching of antidepressants: influence of patient-physician communication. J Am Med Assoc. 2002;288(11):1403-9. https://doi.org/10.1001/jama.288.11.1403.

18. Masand PS. Tolerability and adherence issues in antidepressant therapy. Clin Ther. 2003;25(8):2289-304. https://doi.org/10.1016/S0149-2918(03)80220-5.

19. Bennabi D, Aouizerate B, El-Hage W, Doumy O, Moliere F, Courtet P, et al. Risk factors for treatment resistance in unipolar depression: a systematic review. J Affect Disord. 2015;171:137-41. https://doi.org/10.1016/j.jad.2014. 09.020 .

20. Porcelli S, Drago A, Fabbri C, Gibiino S, Calati R, Serretti A. Pharmacogenetics of antidepressant response. J Psychiatry Neurosci. 2011 36(2):87-113. https://doi.org/10.1503/jpn.100059.

21. Kemp AH, Gordon E, Rush AJ, Williams LM. Improving the prediction of treatment response in depression: integration of clinical, cognitive, psychophysiological, neuroimaging, and genetic measures. CNS Spectr. 2008;13(12):1066-86. https://doi.org/10.1017/S1092852900017120.

22. Locher C, Kossowsky J, Gaab J, Kirsch I, Bain P. Krummenacher P. Moderation of antidepressant and placebo outcomes by baseline severity in 
late-life depression: A systematic review and meta-analysis. J Affect Disord 2015;181:50-60. https://doi.org/10.1016/j.jad.2015.03.062.

23. Association AP. Association, AP Diagnostic and statistical manual of mental disorders. 1995.

24. Naranjo CA, Busto U, Sellers EM, Sandor P, Ruiz I, Roberts EA, et al. A method for estimating the probability of adverse drug reactions. Clin Pharmacol Ther. 1981;30(2):239-45. https://doi.org/10.1038/clpt.1981.154

25. Hartwig SC, Siegel J, Schneider PJ. Preventability and severity assessment in reporting adverse drug reactions. Am J Hosp Pharm. 1992;49:2229-32.

26. Patel M, Patel V, Tiwari D, Shah VR, Patel D, Chanpa N. Efficacy and safety of escitalopram in first episode of major depressive disorder - a tertiary care Indian center experience. J Depress anxiety. 2018;7:1-5.

27. Pinto C, Trivedi JK, Vankar GK, Sharma PS, Narasimha V. An open-label multicentric study of the tolerability and response to escitalopram treatment in Indian patients with major depressive disorder. J Indian Med Assoc. 2007;105(7):364-8

28. Mao PX, Tang YL, Jiang F, Shu L, Gu X, Li M, et al. Escitalopram in major depressive disorder: a multicenter, randomized, double-blind, fixed-dose parallel trial in a Chinese population. Depress Anxiety. 2008;25(1):46-54. https://doi.org/10.1002/da.20222.

29. Li G, Shen Y, Luo J, Li H. Efficacy of escitalopram monotherapy in the treatment of major depressive disorder: a pooled analysis of 4 Chinese clinical trials. Medicine. 2017;96(39):e8142. https://doi.org/10.1097/MD. 0000000000008142

30. Colonna L, Andersen HF, Reines EH. A randomized, double-blind, 24-week study of escitalopram (10 mg/day) versus citalopram (20 mg/day) in primary care patients with major depressive disorder. Curr Med Res Opin. 2005; 21(10):1659-68. https://doi.org/10.1185/030079905X65484.

31. Wade A, Michael Lemming O, Bang Hedegaard K. Escitalopram 10 mg/day is effective and well tolerated in a placebo-controlled study in depression in primary care. Int Clin Psychopharmacol. 2002;17(3):95-102. https://doi.org/1 0.1097/00004850-200205000-00001.

32. Cipriani A, Santilli C, Furukawa TA, Signoretti A, Nakagawa A, McGuire H, et al. Escitalopram versus other antidepressive agents for depression. Cochrane Database Syst Rev. 2009;2:CD006532.

33. Wade AG, Crawford GM, Yellowlees A. Efficacy, safety and tolerability of escitalopram in doses up to 50 mg in Major Depressive Disorder (MDD): an open-label, pilot study. BMC Psychiatry. 2011;11(1):42. https://doi.org/10.11 86/1471-244X-11-42.

34. Trivedi MH, Rush AJ, Wisniewski SR, Nierenberg AA, Warden D, Ritz L, et al. Evaluation of outcomes with citalopram for depression using measurementbased care in $\mathrm{STAR}^{*} \mathrm{D}$ : Implications for clinical practice. Am J Psychiatry. 2006;163(1):28-40. https://doi.org/10.1176/appi.ajp.163.1.28.

35. Joffe RT, Young LT, Levitt AJ, MacQueen G, Marriott MJ, Robb J. Number of episodes and antidepressant response in major depression. Int J Neuropsychopharmacol. 1999;2(2):111-3. https://doi.org/10.1017/S146114 5799001443

36. Fava M, Rush AJ, Alpert JE, Balasubramani GK, Wisniewski SR, Carmin CN, et al. Difference in treatment outcome in outpatients with anxious versus nonanxious depression: A STAR*D report. Am J Psychiatry. 2008;165(3):34251. https://doi.org/10.1176/appi.ajp.2007.06111868.

37. Mulder RT, Watkins WGA, Joyce PR, Luty SE. Age may affect response to antidepressants with serotonergic and noradrenergic actions. J Affect Disord. 2003;76(1-3):143-9. https://doi.org/10.1016/S0165-0327(02)00083-6.

38. Lopez-Castroman J, Jaussent I, Gorwood P, Courtet P. Suicidal depressed patients respond less well to antidepressants in the short term. Depress Anxiety. 2016;33(6):483-94. https://doi.org/10.1002/da.22473.

39. Fergusson D, Doucette S, Glass KC, Shapiro S, Healy D, Hebert P, et al. Association between suicide attempts and selective serotonin reuptake inhibitors: systematic review of randomised controlled trials. BMJ. 2005; 330(7488):396. https://doi.org/10.1136/bmj.330.7488.396.

40. Isacsson $G$, Rich $C L$, Jureidini J, Raven $M$. The increased use of antidepressants has contributed to the worldwide reduction in suicide rates. Br J Psychiatry. 2010;196(6):429-33. https://doi.org/10.1192/bjp.bp.1 09.076166.

41. Griffiths JJ, Zarate CA Jr, Rasimas JJ. Existing and novel biological therapeutics in suicide prevention. Am J Prev Med. 2014;47(3 Suppl 2):S195203. https://doi.org/10.1016/j.amepre.2014.06.012

42. Ozdilek B. Escitalopram-induced bradycardia in elderly individuals: a case series report. Klin Psikofarmakol Bul. 2015;25(2):183-5. https://doi.org/10.54 55/bcp.20140304090654.
43. Thase ME, Larsen KG, Reines E, Kennedy SH. The cardiovascular safety profile of escitalopram. Eur Neuropsychopharmacol. 2013;23(11):1391-400. https:// doi.org/10.1016/j.euroneuro.2013.05.011.

44. Shinde M, Reddy P, Kulmi M, Goyal C. Efficacy and tolerability of milnacipran and escitalopram: a comparative study among patients of depression. Int J Basic Clin Pharmacol. 2018;7(5):839. https://doi.org/10.18203/2319-2003. ijbcp20181489.

45. Sánchez C, Bergqvist PBF, Brennum LT, Gupta S, Hogg S, Larsen A, et al. Escitalopram, the $\mathrm{S}-(+)$-enantiomer of citalopram, is a selective serotonin reuptake inhibitor with potent effects in animal models predictive of antidepressant and anxiolytic activities. Psychopharmacology (Berl). 2003; 167(4):353-62. https://doi.org/10.1007/s00213-002-1364-z.

46. Zhong H, Haddjeri N, Sánchez C. Escitalopram, an antidepressant with an allosteric effect at the serotonin transporter--a review of current understanding of its mechanism of action. Psychopharmacology (Berl). 2012;219(1):1-13. https://doi.org/10.1007/s00213-011-2463-5.

47. Culpepper L. Escitalopram: A new SSRI for the treatment of depression in primary care. Prim Care Companion J Clin Psychiatry. 2002;4(6):209-14. https://doi.org/10.4088/PCC.v04n0601.

48. Basu A, Chadda RK, Sood M, Kaur H, Kukreti R. A preliminary association study between serotonin transporter (5-HTTLPR), receptor polymorphisms (5-HTR1A, 5-HTR2A) and depression symptom-clusters in a north Indian population suffering from Major Depressive Disorder (MDD). Asian J Psychiatr. 2019:43:184-8. https://doi.org/10.1016/j.ajp.2019.05.028.

49. Mandal T, Bairy LK, Sharma PSVN. Association between functional polymorphisms in serotonin transporter gene (SLC6A4) and escitalopram treatment response in depressive patients in a South Indian population. Eur J of Clin Pharmacol. 2020;76(6):807-14. https://doi.org/10.1007/s00228-02002866-4.

50. Tsai MH, Lin KM, Hsiao MC, Shen WW, Lu ML, Tang HS, et al. Genetic polymorphisms of cytochrome P450 enzymes influence metabolism of the antidepressant escitalopram and treatment response. Pharmacogenomics. 2010;11(4):537-46. https://doi.org/10.2217/pgs.09.168.

51. Rajkumar R, Kashyap VK. Genetic structure of four socio-culturally diversified caste populations of southwest India and their affinity with related Indian and global groups. BMC Genet. 2004;5(1):23. https://doi.org/10.1186/1471-21 56-5-23.

\section{Publisher's Note}

Springer Nature remains neutral with regard to jurisdictional claims in published maps and institutional affiliations.

\section{Submit your manuscript to a SpringerOpen ${ }^{\circ}$ journal and benefit from:}

- Convenient online submission

- Rigorous peer review

- Open access: articles freely available online

- High visibility within the field

- Retaining the copyright to your article

Submit your next manuscript at $>$ springeropen.com 